\title{
The Mu2e experiment at Fermilab
}

\author{
Aji Daniel ${ }^{* \dagger}$ \\ University of Houston \\ E-mail: ajidaniel.uh@gmail.com
}

The Mu2e experiment at Fermilab will search for charged lepton flavor violation via the coherent neutrino-less conversion of muons to electrons in the field of an aluminum nucleus. The Mu2e experiment aims to improve the sensitivity by four orders of magnitude over exisiting limits of the branching ratio for this conversion. Mu2e offers discovery potential over a wide array of new physics models and probes mass scales well beyond the reach of present, or future collider, experiments. The Mu2e experimental setup, design and current status is discussed.

XIII International Conference on Heavy Quarks and Leptons

22-27 May, 2016

Blacksburg, Virginia, USA

\footnotetext{
* Speaker.

${ }^{\dagger}$ On behalf of the Mu2e collaboration
} 


\section{Overview}

A neutrino and a charged lepton form a left-handed doublet in the Standard Model (SM), and are assigned a flavor quantum number. The observation that neutrinos oscillate between flavors and thus have mass, means that lepton number is not conserved in the neutral lepton sector and this in turn means that lepton number is also not conserved in charged lepton interactions. However, even assuming massive neutrinos, charged lepton flavor violation (cLFV) processes are extremely suppressed in the standard model. For example, the SM constraint on the branching ratio (BR) of $\mu \rightarrow e \gamma$ is $\leq 10^{-52}$. Most extensions of SM predict rates for cLFV which are within the reach of the next generation of experiments (see reviews [1], [2]). These include models of extra dimensions, GUTs, heavy neutrinos, lepto-quarks, supersymmetry, etc. Thus, an experimental observation of cLFV would be unambiguous evidence of new physics beyond the SM (BSM), and particle physics experiments searching for cLFV are amongst those with highest priority.

Muon to electron conversion is a cLFV process where a muon in the field of a nucleus coherently converts to an electron. Such searches have a long history as is evident from Figure 1. Since the 1940's, the experimental sensitivity has substantially improved. Presently the most stringent limits come for $\mu \rightarrow e \gamma$ from the MEG experiment ( BR $<4.2 \times 10^{-13}$ at $90 \%$ confidence level ) [3] and for muon conversion on gold $\left(R_{\mu e}<6 \times 10^{-13}\right)$ [4].

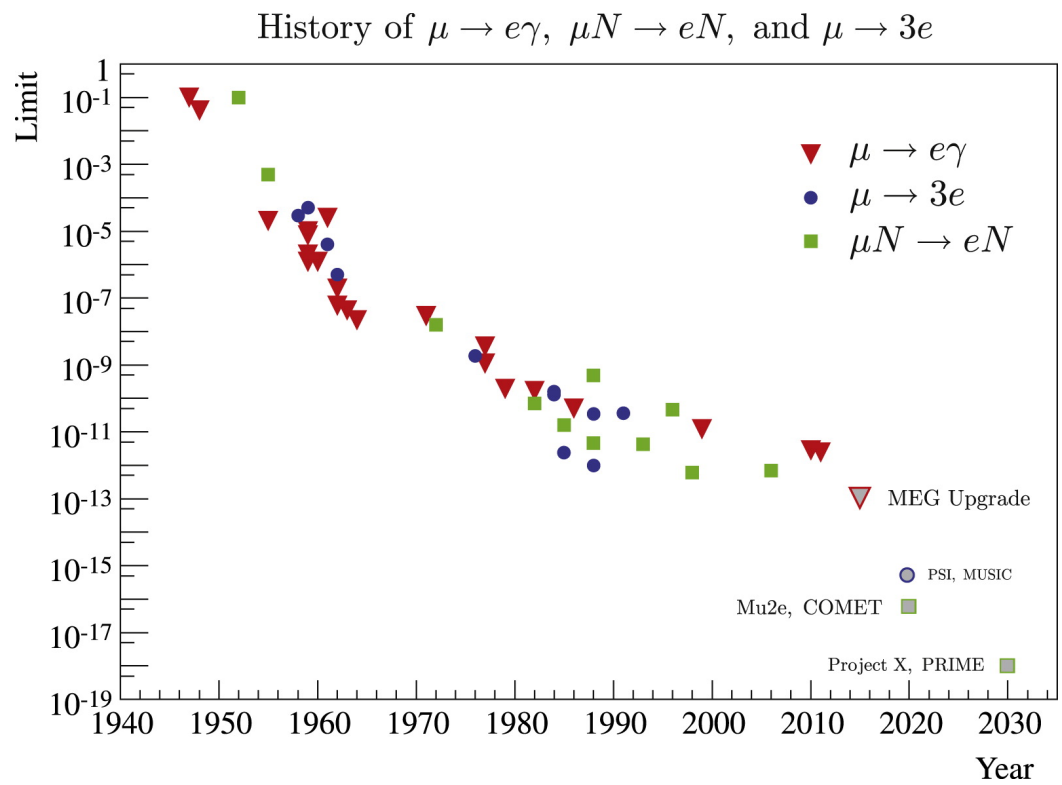

Figure 1: The history of cLFV searches in the muon sector. Figure also shows the experimental sensitivity for the future experiments. From [2]. Many of the new physics models at the weak scale generically imply cLFV rates of about $10^{-15}$, within the reach of Mu2e experiment.

The $\mu \rightarrow e$ process is sensitive to both muon decay to an electron, and 'point interactions' where the leptons and quarks couple through a high mass particle. A model independent description of the cLFV transitions for BSM physics can be expressed by an effective Lagrangian [5], where the different processes can be characterized by dipole amplitudes and contact term operators, respectively. The coefficients of the two operator types are parametrized by two independent constants where $\Lambda$ is the effective mass scale of potentially new BSM physics and $\kappa$ is a dimension-less 
parameter governing the relative contribution of the two operators. A dipole-type operator dominates when $\kappa \ll 1$, while a four-fermion operator dominates when $\kappa \gg 1$. The $\mu \rightarrow e \gamma$ process is mainly sensitive to the dipole amplitude. However, four fermion interactions also contribute to $\mu \rightarrow e N$ and $\mu \rightarrow 3 e$ processes. Figure 2 shows the parameter space as a function of $\kappa$. From the figure it is clear that the sensitivity of $\mu \rightarrow e \gamma$ process is limited to small values of $\kappa$. However, the $\mu \rightarrow e$ conversion experiments can probe new physics over a larger range, but are most sensitive for large $\kappa$. Still measurements with different processes are required to elucidate BSM physics. In summary, Mu2e probes mass scales up to $10^{4} \mathrm{TeV}$ at large values of $\kappa$.

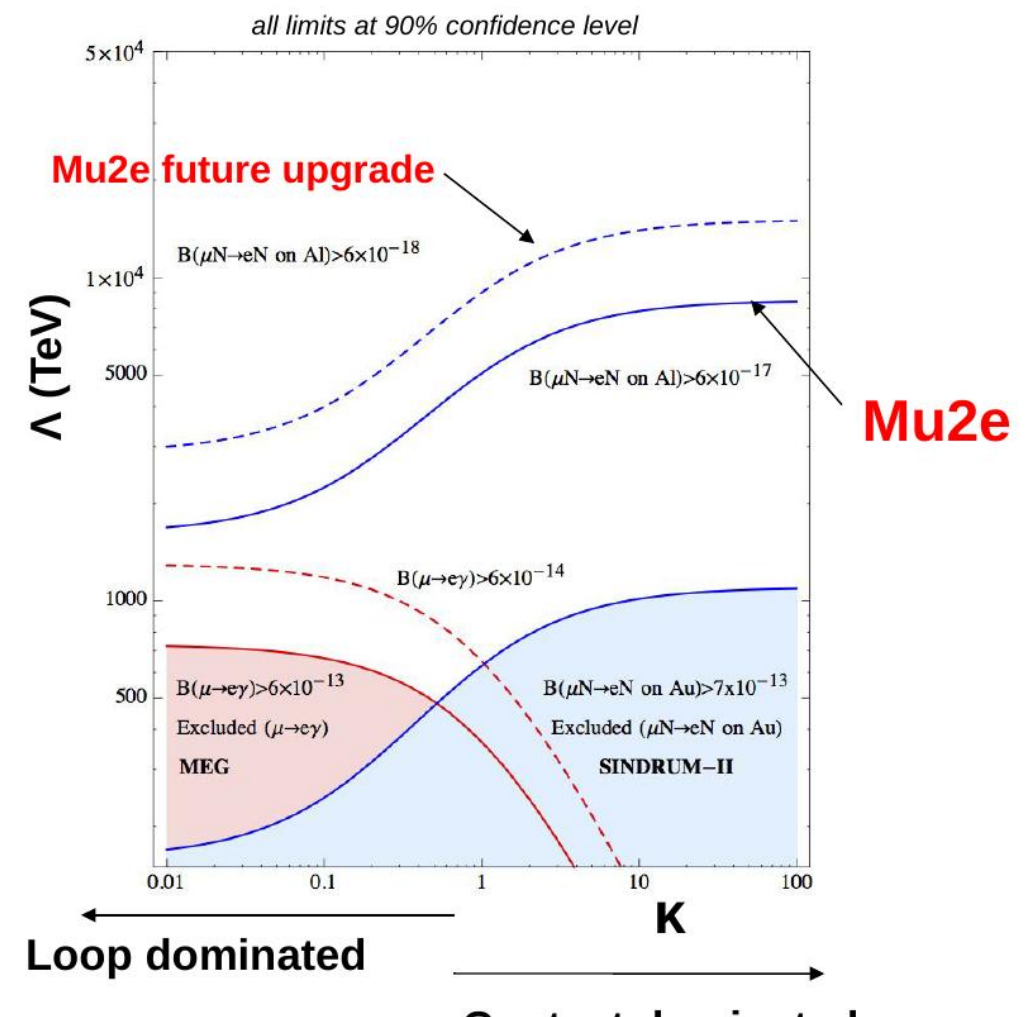

Contact dominated

Figure 2: Sensitivity of cLFV processes to the new physics scale $\Lambda$ as a function of $\kappa$. Also shown are the currently excluded regions of this parameter space by different experiments.

The Mu2e experiment will be undertaken at Fermilab and will attempt to observe neutrinoless, $\mu \rightarrow e$ conversion in the field of $\mathrm{Al}$ nucleus. Thus a negative beam of muons is produced and stopped in an $\mathrm{Al}$ target, and captured into atomic orbits where they form muonic atoms. They then transition to the $1 \mathrm{~s}$ ground state by X-ray emission where they either undergo decay-in-orbit, DIO, $\mu \rightarrow e+v$, or interact coherently with the $\mathrm{Al}$ nucleus to transition to an electron without neutrino emission. In $\mu \rightarrow e$, the emitted electron will have an energy essentially equal to that of the muon mass less the energies of the $\mathrm{Al}$ recoil and atomic binding. For an $\mathrm{Al}$ target, the energy is approximately $105 \mathrm{MeV}$. Background is due to DIO which has a spectrum similar to Michel decay of free muons. This spectrum peaks at $53 \mathrm{MeV}$ and has a small tail extending to the kinematic end point due to nuclear recoil (as seen in Figure 4). Overall, the DIO electron spectra 
falls approximately as $\left(E_{c o n v}-E_{e}\right)^{5}$ [6]. Thus, the main irreducible background for the experiment is caused by the electrons originating from muons bound in the stopping target and decaying in orbit. As no real coincidences are required to signify a signal, $\mu \rightarrow e$ can accept very high beam rates. The Mu2e branching ratio is then defined as the number of $\mu \rightarrow e$ events relative the number of muon captures.

$$
R_{\mu e}=\frac{R\left(\mu^{-}+A(Z, N) \rightarrow e^{-}+A(Z, N)\right)}{R\left(\mu^{-}+A(Z, N) \rightarrow v_{\mu}+A(Z-1, N)\right)}
$$

The Mu2e goal is to achieve a single conversion electron event sensitivity of $2.5 \times 10^{-17}$ or constrain the value of $R_{\mu e}$ below $6 \times 10^{-17}$ at $90 \%$ confidence level.

\section{The Mu2e experiment}

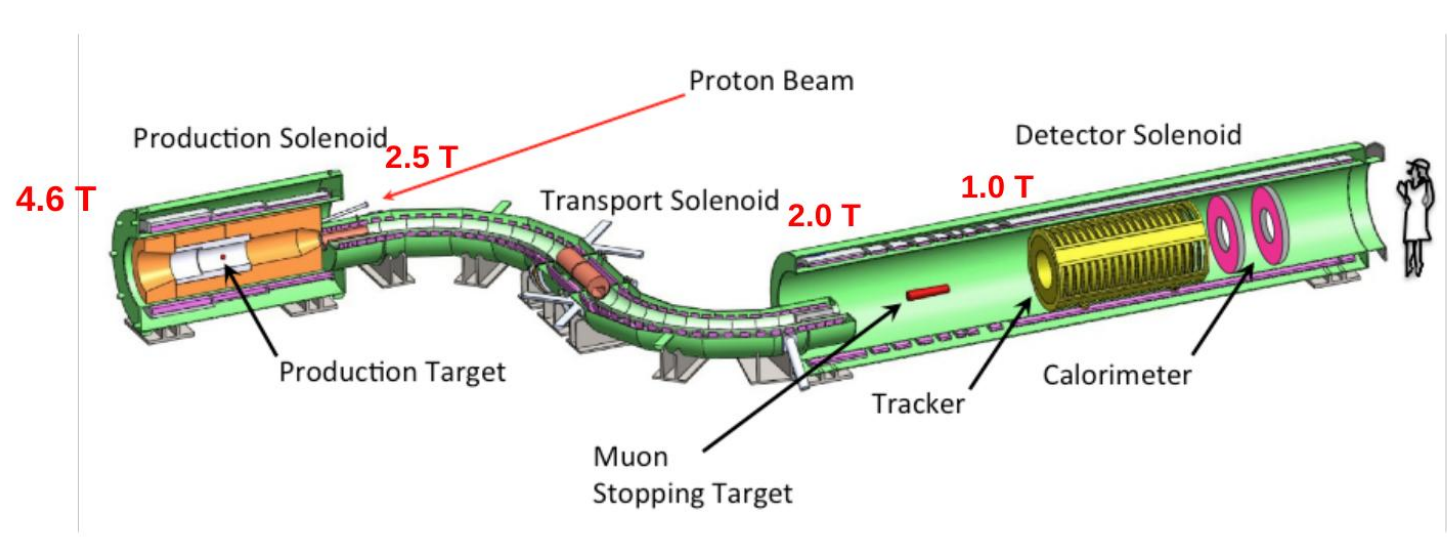

Figure 3: Overview of the Mu2e experiment.

An overview of the Mu2e experiment is shown in Figure 3. Detailed information of the instrumentation can be found in the technical design report [7]. The main components of Mu2e experiment consist of three large superconducting solenoids connected in series; a Production Solenoid (PS), a Transport Solenoid (TS) and a Detector Solenoid (DS). The beam line in the solenoids are evacuated.

Muons for Mu2e are created in the PS by decay pions which are produced in a tungsten target by an $8 \mathrm{GeV}$ pulsed proton beam from Fermilab booster. This beam is bunched into $200 \mathrm{~ns}$ wide time bins which are separated by $1.7 \mu \mathrm{s}$. The beam pulse and Al stopping target are selected to take advantage of the lifetime of a muon atomic orbit in $\mathrm{Al}$ (864 ns), which allows detector activation only between beam spills. This greatly suppresses beam related backgrounds. However, it is necessary to suppress protons in the beam outside of a beam pulse at a level of $10^{-10}$ so that various particles produced in the PS out of the beam timing pulse cannot produce background in the DS. This is mainly handled by an AC dipole, which sweeps out-of-time protons into a collimator. Proton extinction is monitored by dedicated systems. In addition, the magnetic field in the PS increases from 2.5 to $4.6 \mathrm{~T}$ in the same direction of the incoming proton beam and opposite to the outgoing muons beam direction. The gradient field acts as a magnetic lens and push low-energy, charged particles into TS. 
The TS transports low energy muons through the s-shaped solenoid to the DS. A D-shaped collimator is located near its mid-section to preferentially select low-momentum, negative muons. The DS contains the stopping target, and detectors for tracking and energy measurement. It is a 9 m-long, $1 \mathrm{~m}$-radius solenoid, with graded magnetic field from $2 \mathrm{~T}$ to a constant $1 \mathrm{~T}$ in the electron tracker region. The stopping target consists of thin Al disks, and is located in the front section of DS. The gradient field in the upstream region of DS reflects backward emitted conversion electrons improving the acceptance.

The main detector is a low-mass precision straw-tracking detector, which is located in the 1 T, constant field region of the DS. The 4 m long tracker consists of over 20000 straw drift tubes arranged in 18 stations. Each straw tube is $5 \mathrm{~mm}$ in diameter and contains a $25 \mu \mathrm{m}$ sense wire. These are oriented transverse to the axis of the solenoid. The Mylar straw tubes have $15 \mu \mathrm{m}$ thick walls and are filled with an 80-20 Argon- $\mathrm{CO}_{2}$ gas mixture. The tracking-detector determines the momenta of charged particles from their reconstructed, helical trajectories. The central bore of the detector is completely un-instrumented, and allows free passage of particles with momenta less than $55 \mathrm{MeV}$, allowing the majority of DIO electrons to pass undetected through DS.

An electromagnetic calorimeter system composed of two disks of scintillating crystals is placed downstream after the tracker. Each disk contains about 670 CsI crystals and are read out by SiPMs. Similar to the tracker, the electromagnetic calorimeter contains a cylindrical inner hole so it is insensitive to low momentum particles. The calorimeter has a timing resolution of about $500 \mathrm{ps,}$ an energy resolution of about $5 \%$ for $105 \mathrm{MeV}$ electrons, and a position resolution of about $1 \mathrm{~cm}$. The electromagnetic calorimeter contributes to the discrimination between muons and electrons, provides fast timing signals, and a seed for tracking in a complicated reconstruction environment.

After DIO, backgrounds from particles induced by cosmic rays are an important background source for Mu2e. They produce false signals when interacting with detector materials with rates of approximately one per day. To suppress this background, the DS and TS regions of Mu2e are covered by an active Cosmic Ray Veto System (CRV). The CRV consists of multiple layers of overlapping scintillator panels, arranged across the top and sides of DS and downstream half of the TS. A majority coincidence, allows cosmic rays traversing the DS to veto DS detector signals with 99.99\% efficiency.

\section{Conversion signal and backgrounds}

A high precision Germanium Detector will be used to monitor to the atomic X-rays emitted from the stopping target and is important for the normalization of the conversion rate, $R_{\mu e}$. Low energy photons, neutrons and protons are also emitted as a result of the nuclear capture. These constitute an environmental background for the experiment which degrade the efficiency of electron tracking, produce radiation damage in the detection systems. The rates and spectra of these nuclear capture products are being studied by the AlCap experiment at PSI [8].

Figure 5 shows the reconstructed momentum spectrum from a full GEANT4 simulation of Mu2e detector. Simulations assume a conversion rate of $R_{\mu e}=10^{-16}$ and are generated according to the design luminosity of $3.6 \times 10^{20}$ protons on target and $6.7 \times 10^{17}$ stopped muons. The tail on the low-energy side of the $\mu \rightarrow e$ peak is due to multiple scattering of the electrons with materials in stopping target and proton absorber. Mu2e simulations expects an average of about 


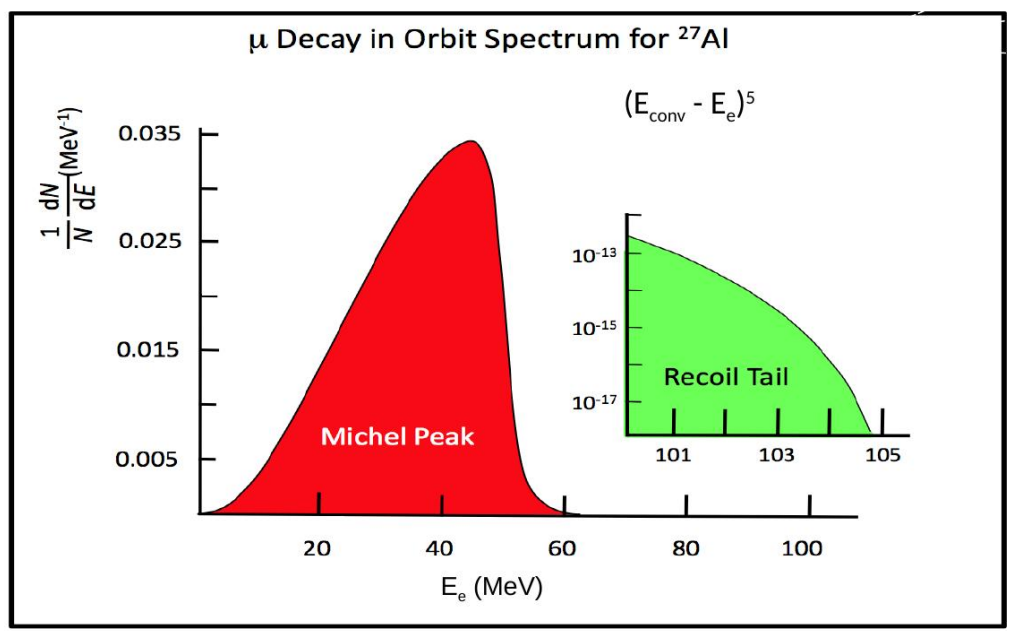

Figure 4: Energy spectra of electrons produced from muon decay in orbit. The inset shows the tail of the DIO electrons on the high end side of the spectrum close to the endpoint energy.

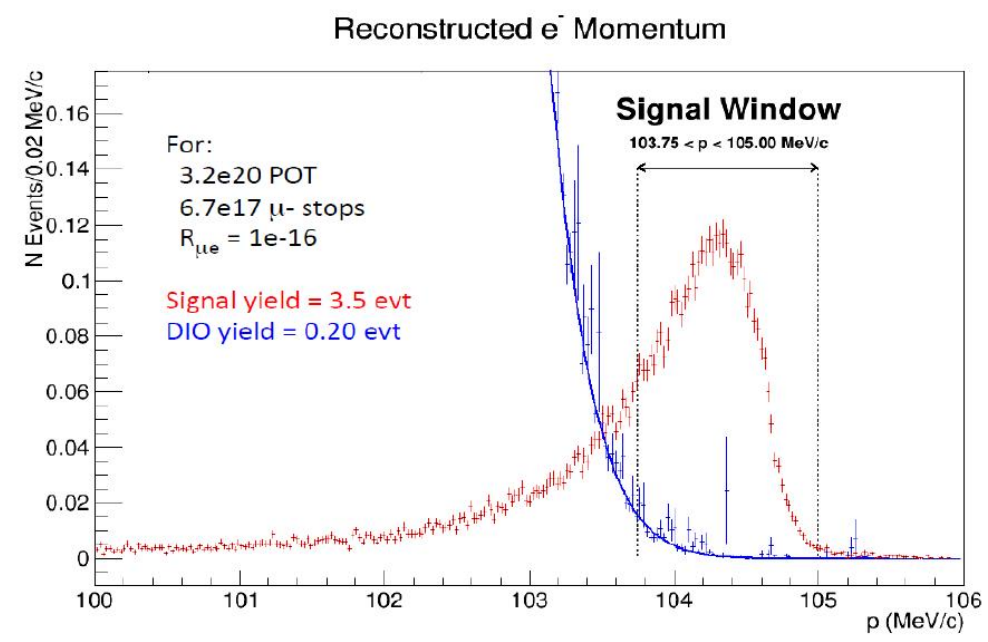

Figure 5: Reconstructed momentum spectra of DIO (blue) and conversion electrons (red) from full simulations performed for the design luminosity and assuming $R_{\mu e}=10^{-16}$.

3.5 conversion electrons in the reconstructed momentum window above $103.75 \mathrm{MeV}$. In the same selection window, an average of 0.2 DIO events and a similar number of background events from a combination of all other source is expected.

\section{Summary}

The Mu2e experiment is expected to probe cLFV with a single event sensitivity of $2.5 \times 10^{-17}$ or to set an upper limit on the conversion rate $R_{\mu e} \leq 6 \times 10^{-17}$ at $90 \%$ confidence level. This would improve the current limit by a factor of 10,000. Absence of a signal also puts severe constraints on the proposed models which address BSM physics. The Mu2e experiment design and construction is 
well underway. R\&D and civil construction phase of the experiment is mostly done. A calorimeter prototype module was tested in an electron beam and shown to have sufficient energy and time resolution. Several straw tracker prototypes have been built, both to test mechanical construction as well as to measure performance with cosmic rays and proton beam tests. Construction is expected to be completed in 2021 , followed by a 3 year physics run.

In the future, the potential use of the Mu2e apparatus with a higher intensity proton beam is being explored with the goal of increasing the sensitivity by an additional factor of 10 . This not only requires a higher beam intensity but also modifications to shielding of the detector.

\section{Acknowledgments}

We would like to thank Fermilab staff for their support. This work is supported by US Department of energy grant number DE-SC0011598.

\section{References}

[1] Kuno, Y. and Okada, Y. Rev. Mod. Phys 73, 151 (2001).

[2] Bernstein, R. and Cooper, P. Phy. Rept 532, 27 (2013).

[3] Baldini, A. M. et al. Eur. Phys. J. C76, 434 (2016).

[4] Bertl, W. et al. Eur. Phys. J. C47, 337 (2006).

[5] Gouvea, A. De and Vogel, P. Prog. Part. Nucl. Phys 71, 75 (2013).

[6] Czarnecki, A., Tormo, G., and Marciano, W. Phy. Rev. D 84, 013006 (2011).

[7] Bartoszek, L. et al. Mu2E Technical Design Report, Fermilab-TM-2594, arXiv:1501.05241 (2015).

[8] AlCap collaboration, http://muon.npl.washington.edu/exp/AlCap/ 